\title{
A Study on Peanut Lectin-Binding Sites in Mucoepidermoid Tumors of the Oral Cavity
}

\author{
by \\ Takashi IWASE, Hideki TSUCHIYA, Yoshiaki MATSUMOTO, \\ Naomichi SANUKI, Eiji SATO and Takeo WADA
}

\section{Introduction}

Lectins, protein and glycoproteins derived from both plants and animals have a unique ability to bind specific sugar molecules and are employed in histochemical studies regarding mucous substances in rat and human intestines.

Some lectins are known to bind various blood group substances. Ulex eurcpenus (UEA 1), for example, binds the $\mathrm{H}(\mathrm{O})$ blood group substance [1].

Recent studies have shown peanut lectin (PNA) to have a specificity for $\beta$-DGal(1-3)-GalNAc and to provide antigenic determination of the $\mathrm{T}$ blood group substance (Tag) [2].

The Tag is present on the surface of red blood cells and lymphocytes, and is covered with terminal sialic acid (so-called cryptic Tag) $[3,4]$.

Numerous studies have examined the prognostic significance of the deletion of the ABH blood group substance in human carcinomas [5,6]. More recently interest has increased in studying the expression of the Tag in human malignant neoplasms.

Expression of the Tag has been examined in carcinomas of the intestine [7,8], urinary bladder [9] and breast [10,11] and the results have shown that the Tag can be expressed from malignant epithelium but is lacking in most normal tissue cells.

There is, however, no information on the localization of the Tag in carcinomas of the oral cavity.

In this study we examined the localization of the Tag in mucoepidermoid tumors by staining paraffin sections with FITC conjugated PNA and reported our findings in correlation with neoplastic changes and the expression of the Tag in comparison with normal salivary glands, sialadenitis and epidermoid carcinomas.

\section{Materials and Methods}

The specimens selected for use were 9 surgical mucoepidermoid tumors (4 gingiva, 3 buccal musoca, 1 palatine mucosa and 1 floor of the oral cavity) in addition to 4 salivary glands ( 2 parotid, 1 submandibular and 1 palatine) and 3 cases of sialadenitis (2 palatine and 1 labial glands) and 5 epidermoid carcinomas (3 gingiva and 2 buccal musoca).

岩瀬孝志，土屋英機，松本芳明，佐貫直通，佐藤栄司，和田武郎：--Department of Pathology, Nihon University School of Dentistry, 1-8-13, Surugadai Kanda, Chiyoda-ku, Tokyo 101, Japan. (Director: Prof. Shinichiro UMEMURA). 
All the specimens were fixed in buffered formalin and embedded in paraffin. We employed the fluorescence method using FITC conjugated PNA(FITC-PNA) for localization of the Tag.

The staining steps are summarized as follows; $0.5 \mu \mathrm{m}$ paraffin sections were deparaffinized in xylol and rehydrated in graded alcohol and washed in PBS (pH 7.2).

Subsequently, the sections were incubated in FITC-PNA (E.Y. laboratory) for 30 minutes at room temperature and washed for 15 minutes in PBS.

After the slides were overslipped with PBS-glycerine the patterns of fluorescence were performed with a Nikon fluorescent microscope using a BG 12 exciting filter and a 530 barrier filter.

For examination of the cryptic Tag, selected sections were incubated in neuaminidase type V (Sigma Chem. Co.) at $0.2 \mathrm{unit} / \mathrm{ml}$ in tris buffer $(\mathrm{pH} \mathrm{5.0)}$ for one hour at room temperature before incubation with FITC-PNA.

\section{Results}

The mucous producing cells (mucicarmine positive cells) of the mucoepidermoid tumors displayed an intense fluorescence for the Tag (Fig. 1). However, the fluorrescence was never observed in the epidermoid cells.

The mucous materials and mucous producing cells in the cystic cavity displayed an intense fluorescence for the cryptic Tag, where they did not show the fluorescence without neuraminidase pretreatment (Fig. 2).

Furthermore, the pretreated tissue sections showed the cryptic Tag on the epidermoid cell surfaces of the mucoepidermoid tumors (Fig. 3).

The epidermoid cell surfaces of the epidermoid carcinomas, however, did not display the fluorescence for the cryptic Tag.

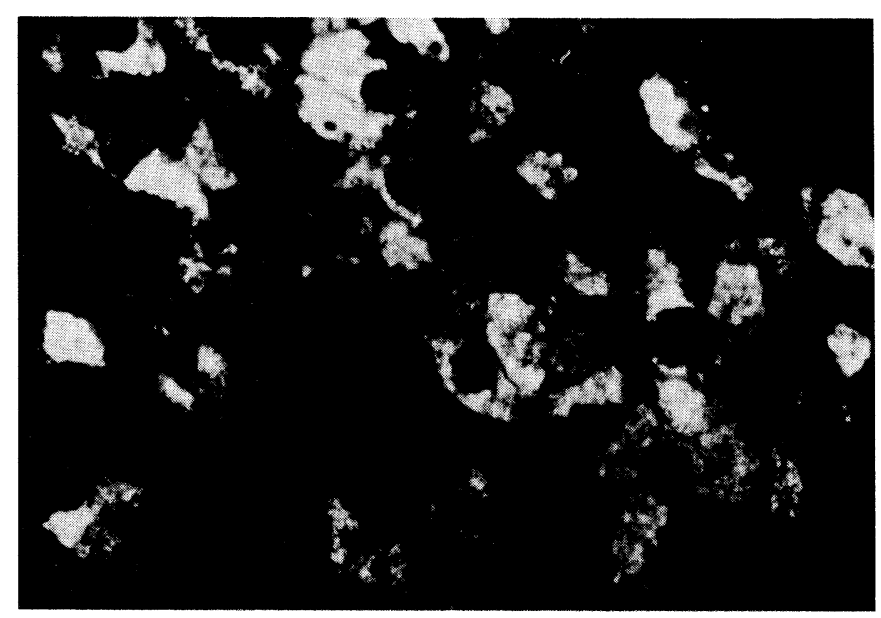

Fig. 1 Mucous producing cells of a mucoepidermoid tumor showing the Tag. 


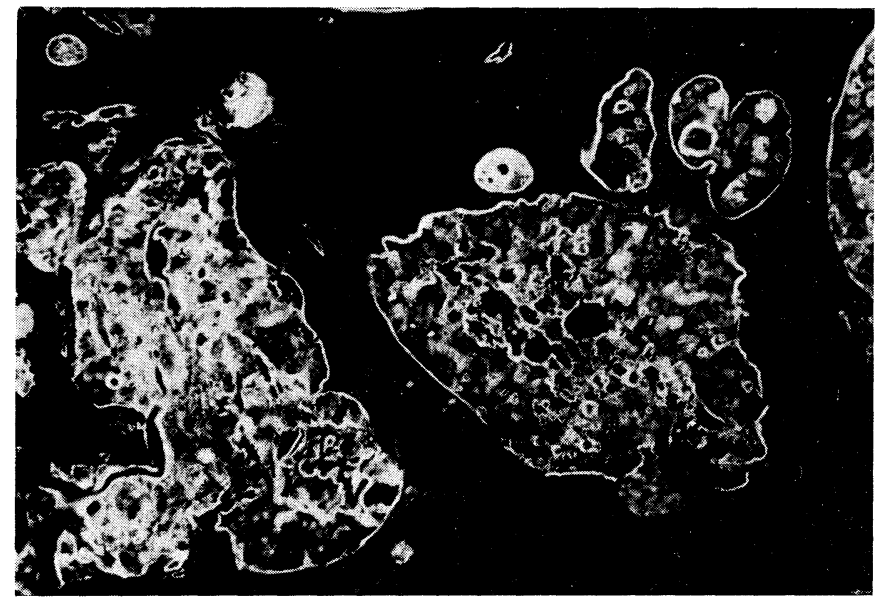

Fig. 2 Expression of the cryptic Tag in mucous materials and mucous producing cells of a mucoepidermoid tumor.

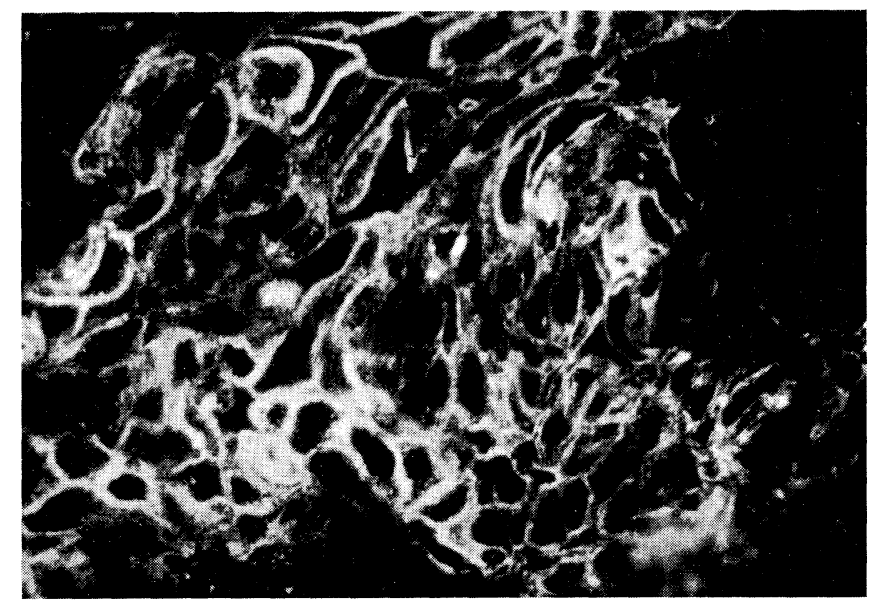

Fig. 3 Expression of the cryptic Tag on the epidermoid cell surfaces of a mucoepidermoid tumor.

Some of the mucous acinar cells in the sialadenitis showed positive staining for the Tag (Fig. 4).

Normal salivary glands had no PNA binding sites, but the serous acinar cells showed PNA binding sites only after pretreatment with neuraminidase.

\section{Discussion}

Recently, the relationship between neoplastic changes and the expression of the Tag in carcinomas of the intestine [7,8], urinary [9] bladder and breast [10,11] have been examined. 


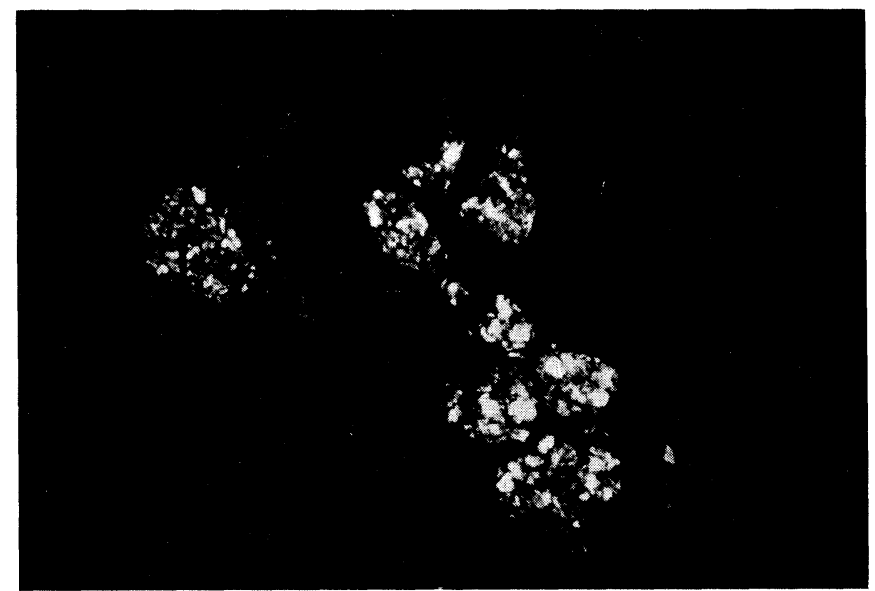

Fig. 4 Mucous acinar cells in sialadenitis (palatine gland) showing the Tag.

It was observed that the malignant neoplastic tissues showed the expression of the Tag whereas normal tissues did not.

Howard [10] and CoON [9] suggested that well differentiated tumors show the expression of the Tag whereas poorly differentiated tumors are cryptic Tag positive.

However, the localization of the Tag in oral carcinomas was not examined, therefore, we examined the expression of the Tag in mucoepidermoid tumors.

PNA has been shown to bind $\beta$-D-Gal(1-3)-GalNAc and Gal. It is considered that the PNA binding sites are due to the Tag in the tissue sections because binding to $\beta$-D-Gal(1-3)-GalNAc has a 50 fold greater affinity than Gal alone [8].

In this study we showed that the mucous producing cells of mucoepidermoid tumors and the mucous acinar cells of sialadenitis display the fluorescence for the Tag.

Regarding the expression of the Tag in colonic carcinomas and the mucosa adjacent to colonic carcinomas, some people consider that the expresison of the Tag in neoplastic tissues shows a malignant change, whereas others consider it to be a reactive phenomenon to an adjacent tumor mass lesion $[12,13]$.

Our observation showed that the expression of the Tag was not due to malignant changes only, as DAwSON [12] and IsSACSON [13] have suggested, because the mucous acinar cells in the sialadenitis also showed the Tag.

Although BoLAND [8] and KLEIN [3] failed to express the Tag and the cryptic Tag in normal colon, COOPER [7] reported the localization of the cryptic Tag in normal epithelium of the colon. COOPER explains that human colonic sialic acid may be very resistant to digestion by neuraminidase pretreatment. However, we were able to demonstrate the expression of the cryptic Tag in serous acinar cells of normal salivary glands after the tissue sections were pretreated with neuraminidase, confirming the studies by Boland [8] and KLEIN [3].

In this study, because the faint fluorescence revealed the cryptic Tag on the epidermoid cell surfaces of the epidermoid carcinomas, we suspect the expression 
of the cryptic Tag on the epidermoid cell surfaces to be characteristic in mucoepidermoid tumors.

\section{Summary}

Peanut lectin (PNA) is known to bind $\beta$-D-Gal(1-3)-GalNAc which provides an antigenic determination for the $\mathrm{T}$ blood group antigen (Tag). Recently, there has been interest in the expression of the Tag in human malignant tumors. We examined 9 mucoepidermoid tumors, 4 normal salivary glands, 3 cases of sialadenitis and 5 epidermoid carcinomas for their ability to express the Tag by staining paraffin sections with FITC conjugated PNA.

Consequently, the mucous producing cells of the mucoepidermoid tumors and the mucous acinar cells of sialadenitis showed the expression of the Tag but the fluorescence was never observed in normal salivary glands. Mucous materials, mucous producing cells in the cystic cavity and the epidermoid cell surfaces of the mucoepidermoid tumors and serous acinar cells of normal salivary glands showed the Tag after the tissue sections were pretreated with neuraminidase, but we never observed the intense fluorescence on the epidermoid cell surfaces of the epidermoid carcinomas. In this study, we suspect that the presence of the Tag in mucoepidermoid tumors does not necessarily mean malignancy.

\section{References}

[1] Gazai, P. and Lalaukie, M.: 1952. Recherches sur quelques phytoagglutininic specifiques groups sanqunis ABO, Acta Haematol., 8, 73-80.

[2] Lotan, R., Skutelsky, E., Danon, D. and Sharon, N. 1975. The purification, composition and specificity of the anti-T lectin from peanut (arachis hypogaea), J. Biol. Chem., 250, 85188523 .

[3] Klein, P. J., Newman, R. A., Muller, P., Uhlenbruck, G., Schaefer, H. E., Lennartz, K. J. and FISCHER, R. 1978. Histochemical methods for the demonstration of THOMSENFRIENDENRICH antigen in cell suspension and tissue section, Klein Wochenshr., 56, 761769.

[4] Reisner, Y.-B. M., Rosenthal, E., Sharon, N. and Ramot, B. 1979. Interaction of peanut agglutinin with normal human lymphocyte and leukemic cells, Proc. Natl. Acad. Sci. USA., 76, 447-453.

[5] Gupta, R. K. and Schuster, R. 1973. Isoantigens A, B and H in benign and malignant lesion of the breast, Amer. J. Pathol., 72, 253-260.

[6] Cooper, H. S. and Haesler, Jr. W. E. 1978. Blood group substance as tumor antigens in the distal colon, Amer. J. Clin. Pathol., 69, 594-598.

[7] Cooper, H. S. 1982. Peanut lectin-binding sites in large bowel carcinoma, Lab. Invest., 47, 594-598.

[8] Boland, O. P., Montgomery, C. K. and KIM, Y. S. 1982. Alternations in human colonic mucin occurring with cellular differentiation and malignant transformation, Proc. Natl. Acad. Sci. USA., 79, 2051-2055.

[9] Coon, T. S., Weinstein, R. S. and Summer, J. L. 1982. Blood group precursor T-antigen expression in human urinary bladder carcinoma, Amer. J. Clin. Pathol., 77, 692-699.

[10] Howard, D. R., Ferguson, P. and Batsakis, J. G. 1981. Carcinoma-associated cytostructural antigen alternation, Cancer, 47, 2872-2877.

[11] Walker, R. A. 1982. Mucoid carcinomas of the breast: A study using mucin histochemistry and peanut lectin, Histopathology, 6, 571-579.

[12] Dawson, P. A. and Filipe, M. I. 1976. An ultrastructural and histochemical study of the mucous membrane adjacent to and remote from carcinoma of the colon, Cancer, 37, 2388-2398. 
[13] Issacson, P. and ATwood, P. R. A. 1979. Failure to demonstrate specificity of the morphological and histological changes in mucosa adjacent to colonic carcinoma (transitional mucosa), J. Clin. Pathol., 32, 214-223. 